\title{
Spatial analysis of nanofluidic-embedded biosensors for wash-free single-nucleotide difference
} discrimination

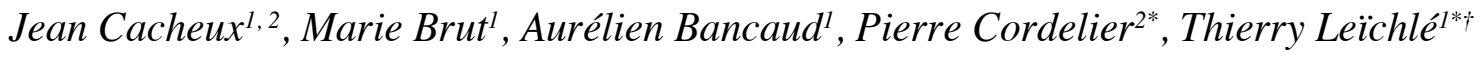

${ }^{1}$ LAAS-CNRS, Université de Toulouse, CNRS, Toulouse, France

${ }^{2}$ Université Fédérale Toulouse Midi-Pyrénées, Université Toulouse III Paul Sabatier, INSERM, CRCT, Toulouse, France

KEYWORDS: Single-nucleotide difference, miRNA, nanofluidics, biosensing, fluorescence, depletion, hybridization

\footnotetext{
* Both authors contributed equally to this work.

$\dagger$ tleichle@laas.fr
} 


\section{ABSTRACT}

In this work, we demonstrate that the analysis of spatially resolved nanofluidic-embedded biosensors permits the fast and direct discrimination of SND (single-nucleotide difference) within oligonucleotide sequences in a single step interaction. We design a sensor with a linear dimension much larger than the channel depth in order to ensure that the reaction over the whole sensor is limited by the convection rate. Thus, the targets are fully collected, inducing a non-uniform spatial hybridization profile. We also use the nanoscale height of the channel, which enables us to minimize the amount of labeled molecules flowing over the sensor and hence to reduce the fluorescence background, to carry out real-time hybridization detection by fluorescence microscopy. Taken together, these design rules allow us to show that the spatial hybridization profile depends on the duplex affinity, and we speculate that the on and off-rate constants can be inferred during target injection, which is not possible in local analysis where the dissociation step through rinsing must be conducted. We finally manage to discriminate a GT mismatch on a microRNA sequence by optimizing the interaction temperature and the probe design after a few minutes of interaction in a single step protocol. This work may be applied to any biosensing transduction scheme with spatial resolution, e.g. surface plasmon resonance imaging, integrated into nanofluidic channels for applications where high oligonucleotide sequence selectivity and short analysis times are required. 
A SND (single-nucleotide difference) can originate from either a polymorphism or a mutation [1] of one base into an oligonucleotide sequence. The gold standard for the identification of such SND is the Next Generation Sequencing [2-3], a costly and time-consuming approach that enables the systematic discovery of new SND. Sanger sequencing that targets specific sequences is another cheaper and faster solution to achieve SND analysis, however, it is not well-suited to short targets, e.g. miRNA, since cloning and amplification steps are carried out before sequencing [4-5]. Following identification, target detection methods using an appropriate probe design, such as DNA microarrays [6], can be relevant. They mostly consist in measuring the ratio of hybridized duplexes while sweeping the temperature in order to detect a reduced melting temperature (the SND sequence is energetically less stable than the full-match target). Nevertheless, these methods always require a pre-amplification step via PCR (polymerase chain reaction) because of their low sensitivity [7-8]. Alternative approaches include the use of specific rolling circle amplification [9], a multi-step protocol based on enzymatic reaction and quantum dots labeling. Additional strategies are based on molecular beacons coupled to quantum dots [10] or graphene sheets [11], both using a specific revelation step and only revealing differences at equilibrium. Also, specific enzymes (T4 RNA ligase and DNAzyme) coupled to gel electrophoresis [12] offer good selectivity but involve multiple preparation steps. Finally, addressing the association and dissociation rates of the duplex formation may help discriminate single base differences [13-14]. It was shown that the discrimination is clear only during the rinsing step because the dissociation rate is much faster when a mismatch is present in the duplex while no discrimination is possible when solely studying the association rate [15].

Despite the fact that highly sophisticated detection methods have already been developed, none of them is compatible with direct detection and absolute quantification of oligonucleotides since 
they often require a cumbersome, multi-step protocol including target amplification. Moreover, such technologies rarely point out specificity at the base level (single nucleotide differences, SND) nor the stability of the SND/probe duplex that strongly relies on the position and the nature of the bases involved in the mismatch [16-17].

Here, we present a novel nanofluidic-embedded biosensor functionalized with DNA probe sequences complementary to candidate oligonucleotides, specific enough to detect SND via affinity discrimination in a single-step real-time detection protocol. Our sensing platform relies on the analysis of the spatial hybridization profile along the sensor, derived through fluorescence imaging, that highly relies on the duplex affinity.

By optimizing the interaction temperature and the probe design, we manage to discriminate goldstandard, hard-to-detect mismatches on a 22 bases single-stranded microRNA sequence within a few minutes of interaction, in the absence of preanalytical processing or a washing step. Taken together, the novel analysis method presented herein not only allows for the real-time identification of microRNA at the single-base level but can also be translated into further applications (DNA, proteins, etc.) with any transduction scheme (surface plasmon resonance, MEMS, etc.) as long as the biosensor is spatially resolved into nanochannels.

RESULTS AND DISCUSSION

\section{Nanofluidic-embedded biosensing platform}

The biosensor consists of a functionalized gold patch embedded in a nanochannel connected to two inlet/outlet microchannels. The nanoscale size of the fluidic channel results in several 
consequences unique to our platform. First of all, the time for molecules to diffuse over the channel height, which is within the millisecond range, is highly shortened in comparison with the travel time by convection over the gold patch. Hence, the sensor operates into a "full target collection" regime where upcoming molecules are consumed by the sensor and a depletion zone forms along the fluid flow (figure 1.a) [18]. Besides, because the sensor length $(50 \mu \mathrm{m})$ is much larger than the channel height $(500 \mathrm{~nm})$, the biochemical reaction over the whole sensor surface is limited by the convection rate. As a result, the reaction rate is not uniform at the sensor surface, unlike what is usually observed in microfluidic devices. By defining a sensor with a high spatial resolution $(<1$ $\mu \mathrm{m})$, it is possible to locally study the molecular interaction and plot the non-uniform reaction rate along the sensor length.

The other consequence of using nanofluidics is that the number of labeled target molecules flowing above the sensor (concentration $\times$ channel thickness) is reduced and the resulting fluorescent background is negligible. Thus, it is possible to observe the dynamic target/probe molecules reactions in real-time and without the need to wash the channel. Consequently, we can use our sensing platform with standard fluorescence microscopy to probe the sensor surface with high spatial resolution (determined by the camera pixel size) and study dynamic interactions with a simple and standard laboratory equipment. 


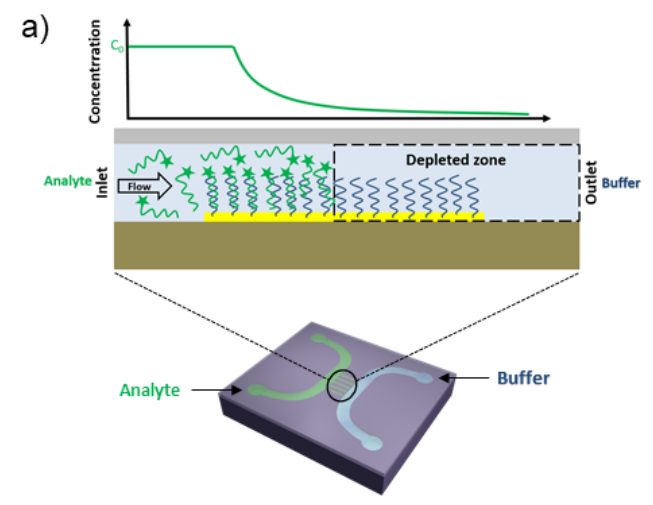

b)

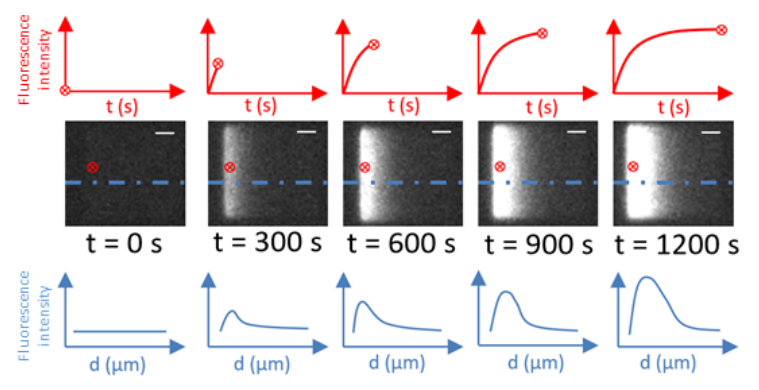

Figure 1. a) Working principle of the nanofluidic biosensor: target molecules (green) injected in the nanochannel are trapped by the specific probes (blue) immobilized onto a gold patch. This results in a depleted zone and the concentration of target molecules decreases along the fluid flow. b) Time lap fluorescence images recorded onto the sensor (scale bar $=10 \mu \mathrm{m}$ ) and data analysis: classical kinetic study is obtained by plotting the fluorescence signal from a spatially resolved specific sensor location (one camera pixel) versus time (top, red), and profile analysis proposed in this work, obtained by plotting the fluorescence intensity along a line of pixels on the sensor versus the distance (bottom, blue).

Using fluorescence images of the biosensor, there are various ways to analyze the signal over the sensor and to derive information on molecular affinity (figure 1.b). The classical approach is based on the spatially localized data, where the fluorescence signal recorded as a function of time results in common kinetic sensorgrams and fitting methods are used to obtain association and dissociation constants. To this aim, the fluorescence signal, i.e. the reacting probe ratio, is measured with a continuous flow of labeled targets. Because the association rate depends on the 
target concentration, fluorescence intensity is taken at the forefront of the sensor where the target concentration above the sensor equates the solution concentration. Moreover, the signal is measured locally using only one (or a few) pixel in order to be in reaction-rate limited vs. convection-limited interaction regime (figure 1.b, top). Then, the sensor is rinsed (by reversing the fluid flow in the nanochannel and allowing buffer solution to flow back on the sensor) to dissociate the probe/target duplexes. Association and dissociation constants are then derived from the kinetic sensorgram using a simple Langmuir fit of the data.

From the same data set, it is also possible to proceed to a global analysis of the sensor by plotting the fluorescence signal along the sensor length following a line of pixels at various times (figure $1 . b$, bottom). Because of the non-uniform reaction kinetics taking place along the sensor length due to the convection-limited regime and the target depletion layer, this analysis provides additional information on the probe/target interaction. Consequently, same association/dissociation constants can be obtained during a single association step without the need for time-consuming rinsing steps.

\section{Hybridization profile analysis}

When biomolecules with strong affinity interact in appropriate flow conditions, target molecules injected in the nanochannels are captured by the sensor until it saturates and the flowing solution is depleted. As a result, the fluorescence profile along the sensor length exhibits a sharp peak at the front and a rapid decay. Considering the same geometry and flow rate, weaker duplex affinities result in lower reaction rates. The direct consequence is that the amount of target molecules captured at the upcoming sensor surface is lower and a portion of injected targets can flow over the sensor and interact downstream. This leads to the leveling of the fluorescence profile where 
the signal peak at the front of the sensor is not as high and the decay, not as abrupt. In the extreme case, where the interaction is non-specific, the reaction rate is low enough leading to a reaction rate uniform all over the sensor surface and so is the profile.

We have experimentally observed the aforementioned behavior and studied the dependence of the hybridization profile on the duplex affinity using microRNA targets. To this aim, we have selected two targets from the let-7 microRNA family that differ only from two nucleotides (hereinafter called "full-match" for the let-7c sequence and "DND", i.e. double nucleotide difference, for the let-7f sequence) and one DNA probe sequence that includes locked nucleic acid (LNA) bases complementary to the full-match sequence (p-let-7c-6LNA sequence, see the supplementary information table S1). First, we studied the kinetics of interaction of each duplex in a classic configuration, where association and dissociation rates are obtained from spatial localized data using the procedure described in the previous section. Since similar affinities were measured at room temperature (see the supplementary information figure S1 a), experiments were conducted at higher temperatures (the affinity of each duplex depends exponentially on the reciprocal of the temperature) to help better discriminate the two targets (figures 2.a and 2.b). It is worth noting that the fluorescence signal could not be directly compared between experiments at different temperatures because the dye yield is temperature-dependent $[19,20]$. Thus, data were normalized using the steady state fluorescence signal of the full-match sequence for each temperature (figures 2.a, 2.b). 

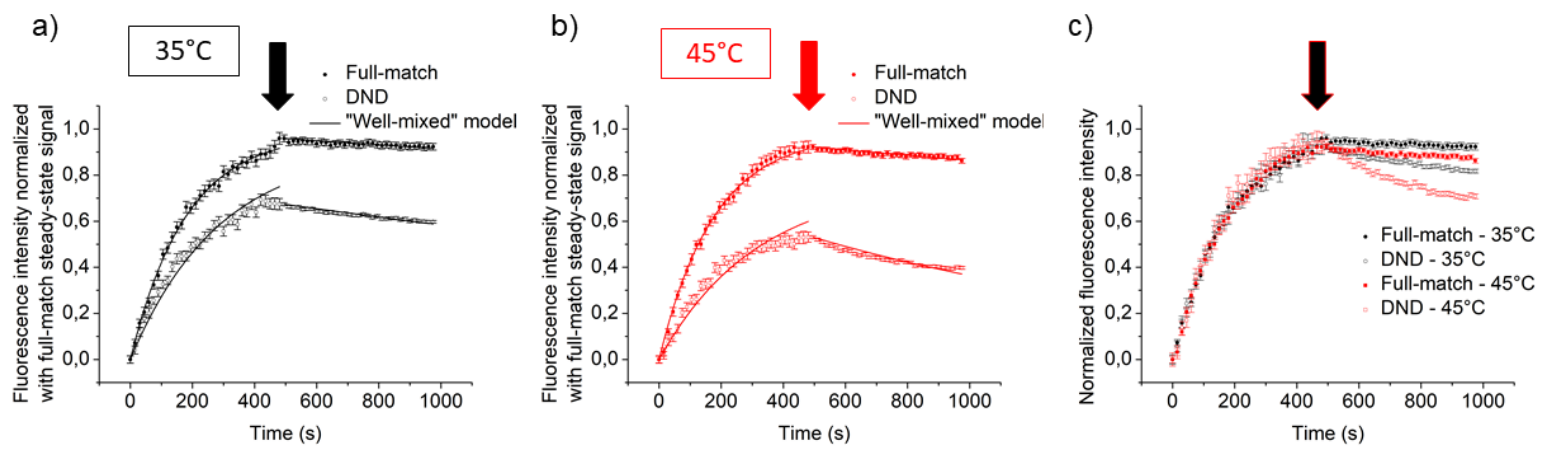

Figure 2. Kinetic sensorgrams of the full-match and DND targets at $35^{\circ} \mathrm{C}$ (a) and $45^{\circ} \mathrm{C}$ (b) after normalizing each set of data using the full-match steady-state value: during these experiments, a $100 \mathrm{nM}$ analyte solution is injected in the nanochannel with a pressure of 10 mbar during $480 \mathrm{~s}$, then the sensor is rinsed with pure buffer by reversing the pressure flow (at the time indicated by the arrow). c) Same data as in (a) and (b) after normalizing each set of data with its steady state value.

Using the full-match target steady-state signal for data normalization, a difference between the plots for the two targets is clearly observed after target-probe interaction, even at $35^{\circ} \mathrm{C}$ (figure 2.a). As expected, better discrimination is obtained by increasing the temperature to $45^{\circ} \mathrm{C}$ (figure $2 . \mathrm{b}$ ). However, experiments conducted at $55^{\circ} \mathrm{C}$ (see the supplementary information figure $\mathrm{S} 1 . \mathrm{b}$ ) show that the DND target is no longer detected (no hybridization occur on the sensor) while a significant fluorescence is still measurable with the full match target. Using the experimental sensorgrams, binding constants were extracted using a Langmuir fit [21, 22], and then experimental Gibbs free energies were derived for each duplex. These experimental data are in very good agreement with 
the theoretical energies obtained with molecular dynamics simulations (see data in table 1 and description of the theoretical data calculation in the supplementary information).

An interesting point is that when the data normalization for full-match and DND is carried out using each steady state value (figure 2.c), no difference is observed during the association step between the signal from the full-match and from the DND targets whatever the operating temperature. Indeed, while the hybridized probe ratio is lower for the DND target, the dynamic behavior of the hybridization is similar. Nevertheless, a clear signal difference is measured during the dissociation step, even after normalization, because the DND unhook much faster than the fullmatch targets. Furthermore, the dissociation rate does not rely on sample concentration whereas the association rate does: this means that if the sample concentration is unknown, it is mandatory to carry out the dissociation step by rinsing the sensor to achieve proper target discrimination in the absence of reference.

\begin{tabular}{|c|c|c|c|c|}
\hline Target & $\begin{array}{c}\text { Interaction temperature } \\
\left({ }^{\circ} \mathrm{C}\right)\end{array}$ & $\begin{array}{c}\Delta \mathrm{G}_{\text {Gibbs th }} \\
(\mathrm{kcal} / \mathrm{mol})\end{array}$ & $\begin{array}{c}\Delta \mathrm{G}_{\text {Gibbs exp }} \\
(\mathrm{kcal} / \mathrm{mol})\end{array}$ & $\begin{array}{c}\text { Error } \\
(\%)\end{array}$ \\
\hline \multirow{2}{*}{$\begin{array}{c}\text { Full- } \\
\text { match } \\
(\text { let-7c) }\end{array}$} & 35 & -12.49 & -12.36 & 1.0 \\
\cline { 2 - 5 } & 45 & -12.23 & -12.18 & 0.4 \\
\hline $\begin{array}{c}\text { DND } \\
\text { (let-7f })\end{array}$ & 35 & -11.39 & -11.36 & 0.2 \\
\cline { 2 - 5 } & 45 & -10.57 & -10.59 & 0.2 \\
\hline
\end{tabular}

Table 1. Experimental and theoretical Gibbs free energies of the two targets and the LNA probe at the working temperatures.

The alternative method to analyze data during the association step, as proposed in this work, is to plot the hybridization profile along the sensor instead of providing a local analysis vs. time. Similar to kinetic analysis, the fluorescence response is first normalized using the steady state 
signal of the full-match target (figures 3.a and 3.b). In addition, and because the flow velocity increases by approximately $20 \%$ due to the change in solution viscosity when shifting temperature, data are collected $360 \mathrm{~s}$ and $300 \mathrm{~s}$ after injecting the sample for experiments performed at $35^{\circ} \mathrm{C}$ and $45^{\circ} \mathrm{C}$, respectively. This ensures an equivalent amount of molecules ( 500 zeptomoles) injected into the sensor. As with the kinetic analysis, a clear signal difference between the two sequences is detectable at $35^{\circ} \mathrm{C}$, because the duplex binding constant is weaker for the DND target. Consequently, the number of hybridized probes at equilibrium is lower and target molecules that do not interact with probes at the beginning of the sensor are carried out by the fluid flow and have the ability to do so downstream. This phenomenon may account for a lower response at the front of the sensor in case of the DND target while the measured fluorescence intensity is higher further down. The interesting feature of this analysis strategy is that when each profile is normalized with its own steady state value (figure 3.c), differences in the spatial profiles of the full-match and DND targets are still clear unlike what is observed on the association part of the sensorgrams (figure 2.c). A COMSOL simulation (fitting method in the supplementary information) using the experimental affinity constants obtained from the sensorgram fits confirms the influence of the differences in affinity on the hybridization profile $(\mathrm{R}$-squared $=0.9903)$. 

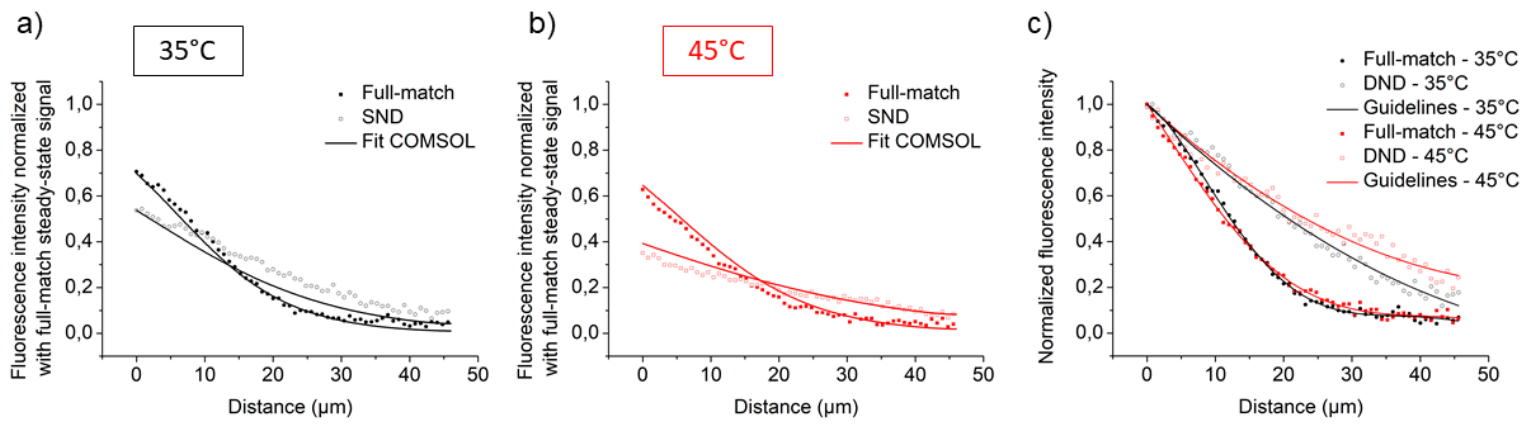

Figure 3. Hybridization profiles recorded during the association step a) at $35^{\circ} \mathrm{C}$ after $360 \mathrm{~s}$ of interaction, b) at $45^{\circ} \mathrm{C}$ after $300 \mathrm{~s}$ of interaction, all normalized using the full-match target steady state signal and c) Same data as in (a) and (b) after normalizing each set of data with its steady state value.

\section{Discrimination of single base difference within microRNA family members}

To demonstrate the high selectivity of our nanofluidic-embedded biosensor platform coupled to the proposed spatial profile analysis, we have selected two microRNA targets very difficult to discriminate, let-7b and let-7c, belonging to the same microRNA family (let-7), which sequences differ only by one nucleotide. The sixth adenine nucleotide (A) from the let-7c sequence is replaced by a guanine base $(\mathrm{G})$ in the let-7b sequence leading to a guanine-thymine mispairing upon hybridization with the let-7c complementary probe (p-let-7c-3LNA), which is one of the most stable mismatch $\left(\Delta \mathrm{T}_{\mathrm{m}} \sim 1^{\circ} \mathrm{C}\right)$. To increase the probe affinity with the RNA targets and improve the selectivity of the probe [23-24], we have designed probes with added LNA nucleotides at the SND position and the two nearby bases (cf. table S1 in the supporting information). Next, the hybridization temperature was optimized using melting curves, during which the ratio of hybridized probe is measured on the sensor at a constant target concentration with increasing 
temperature. Since a slight signal difference between the two target sequences was observed from $50^{\circ} \mathrm{C}$ to $60^{\circ} \mathrm{C}$ (figure $\mathrm{S} 2$ - not seen with classic DNA probes), this temperature range was selected for the hybridization experiments. The resulting hybridization profiles for the full-match and SND sequences at both temperatures are shown in figure 4: while discrimination is tenuous at $50^{\circ} \mathrm{C}$, a difference between the profiles is clearly observed after a few minutes of interaction at $60^{\circ} \mathrm{C}$, demonstrating that, following fine-tuning of the experimental conditions, the spatial analysis of the nanofluidic-embedded biosensor provides means to quickly and selectively distinguish SND in a single-step interaction.

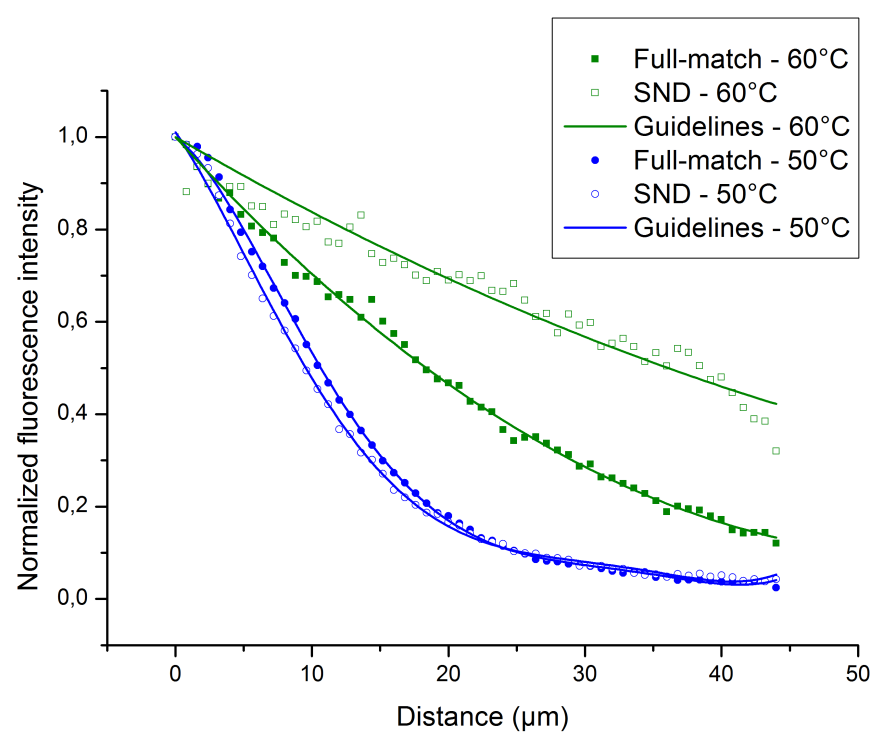

Figure 4. Hybridization profiles recorded after 1200 seconds of interaction at $50^{\circ} \mathrm{C}$ and $60^{\circ} \mathrm{C}$ for the SND and the full-match targets (applied pressure: 5 mbar). 


\section{CONLUSION}

In this work, we present a novel microfluidic setting affording real-time detection of microRNA and selective enough to discriminate these tiny molecules at a single-base level. Moreover, this work describes for the first time a new analysis strategy for affinity biosensors operating in a convection-limited regime and full target collection with spatial resolution. Indeed, the hybridization profile measured at the sensor surface provides additional information compared to classically non-spatially resolved analysis due to the non-uniform hybridization rate along the sensor. Hence, kinetic data can possibly be derived during the association step only since it involves multiple binding and unbinding events along the fluid flow. This analysis stands out from classical kinetic approaches where full sensorgrams obtained not only through association but also dissociation require to rinse the sensor. Additionally, we have demonstrated that, by optimizing the operating temperature and the probe design, we could discriminate one of the most stable SND in a few minutes. Hence, this work opens doors to many applications where high-selectivity and short analysis times are requested. We suggest that it could be a suitable technique to detect DNA mutation or methylation, another landmark of (epi)genetic modification, which seems to modulate DNA duplex affinity [25]. More generally, the presented analysis method can also be applied to any transduction scheme for spatially resolved biosensors integrated into nanofluidic channels.

\section{MATERIALS AND METHODS}

\section{Materials and reagents}

Oligonucleotides (targets and probes) sequences were synthesized by Eurogentec (Liège, Belgium) ( $c f$. sequences in the supplementary table S1). Buffer solution PBS $1 \times$ was purchased 
from Life Technologies (Carlsbad, USA). MCE (mercaptoethanol) was purchased from SigmaAldrich (USA).

\section{Fabrication of bio-functionalized nanofluidic devices}

The chip microfabrication process has been previously described [26]. The functionalization protocol used to graft the probe molecules onto gold patches embedded in the nanofluidic channels was carried out before chip sealing. Briefly, it consists in a one-hour incubation of thiolated DNA probes (p-let7-c-3LNA or p-let7-c-6LNA in table S1) followed by a one-hour incubation of MCE after deionized water rinsing and $\mathrm{N}_{2}$ drying [27]. Encapsulation of the fabricated micro- and nanochannels was carried out by means of a hard-PDMS coated cover glass activated with oxygen plasma at room temperature, as described previously [28].

\section{Fluorescence interaction}

After sensor functionalization and chip encapsulation, fluorescently labeled microRNA targets diluted at $100 \mathrm{nM}$ in PBS $1 \times$ solution were introduced in the nanofluidic channels using pressure driven flow (MFCS, fluigent). Applied pressure during the experiments was 5 or 10 mbar. Binding of the fluorescent targets to the biosensor surface immobilized with probe molecules was monitored in real-time using an inverted fluorescence microscope (Olympus IX71) coupled to a light source (lumencor, SOLA 6-LCR-SB) and an EMCCD (iXon ${ }^{\mathrm{EM}}+885$, Andor) with a 20× objective and a Cy3-dedicated filter set (41007a-Cy3, chroma). The exposure time was $200 \mathrm{~ms}$ and the imaging period was 15 s or $30 \mathrm{~s}$. Reverse buffer flow operation with PBS $1 \times$ was used to achieve full kinetic sensorgrams (association and dissociation phases). Temperature was controlled by the PE94 LinkPad temperature controller from Linkam (UK). 


\section{ASSOCIATED CONTENT}

\section{Supporting Information.}

The following files are available free of charge.

Table of oligonucleotide sequences, Kinetic sensorgrams of the full-match and DND targets at room temperature and $55^{\circ} \mathrm{C}$, On chip melting curves for the SND and the full-match targets, Profile fitting method with COMSOL simulation and Theoretical Gibbs free energy calculation method (PDF).

\section{AUTHOR INFORMATION}

\section{Corresponding Author}

†tleichle@laas.fr

\section{Author Contributions}

The manuscript was written through contributions of all authors. All authors have given approval to the final version of the manuscript.

\section{Funding Sources}

ANR-13-IS10-0001, IDEX Programme Oncodevice Action Thématique Stratégique 2013 Université Fédérale de Toulouse, Fondation ARC programme Jeune Chercheur.

\section{ACKNOWLEDGMENT}

We thank CALMIP supercomputer center for CPU resources. 


\section{ABBREVIATIONS}

SND, single nucleotide difference; DND, double nucleotide difference.

\section{REFERENCES}

1. Karki, R.; Pandya, D.; Elston, R. C.; Ferlini, C. Defining 'mutation' and 'polymorphism' in the era of personal genomics. BMC Med Genomics 2015, 8. DOI 10.1186/s12920-015-0115-z.

2. Nielsen, R.; Paul, J. S.; Albrechtsen, A.; Song, Y. S. Genotype and SNP calling from nextgeneration sequencing data. Nat Rev Genet 2011, 12, 443-451.

3. Li, R.; Li, Y.; Fang, X.; Yang, H.; Wang, J.; Kristiansen, K.; Wang, J. SNP detection for massively parallel whole-genome resequencing. Genome Res 2009, 19, 1124-1132.

4. Sanger, F.; Nicklen, S.; Coulson, A. R. DNA sequencing with chain-terminating inhibitors. Proc. Natl.Acad.Sci. U.S.A., 1977, 74, 5463-5467.

5. Mullikin, J. C.; Hunt, S.E.; Cole, C.G.; Mortimore, B.J.; Rice, C.M.; Burton, J.; Matthews, L.H.; Pavitt, R.; Plumb, W.; Sims, S.K.; Ainscough, R.M.R.; Attwood, J.; Bailey, J.M.; Barlow, K.; Bruskiewich, R.M.M.; Butcher, P.N.; Carter, N.P.; Chen, Y.; Clee, C.M.; Coggill, P.C.; Davies, J.; Davies, R.M.; Dawson, E.; Francis, M.D.; Joy, A.A.; Lamble, R.G.; Langford, C.F.; Macarthy, J.; Mall, V.; Moreland, A.; Overton-Larty, E.K.; Ross, M.T.; Smith, L.C.; Steward, C.A.; Sulston, J.E.; Tinsley, E.J.; Turney, K.J.; Willey, D.L.; Wilson, G.D.; McMurray, A.A.; Dunham, I.; Rogers, J.; Bentley, D.R. An SNP map of human chromosome 22. Nature, 2000, 407, 516-520. 
6. Urakawa, H.; El Fantroussi, S.; Smidt, H.; Smoot, J.C.; Tribou, E.H.; Kelly, J.J.; Noble, P.A.; Stahl, D.A. Optimization of single-base-pair mismatch discrimination in oligonucleotide microarrays. Appl. Environ. Microbiol., 2003, 69, 2848-2856.

7. Upadhyaya, A.; Smith, R. A.; Chacon-Cortes, D.; Revêchon, G.; Bellis, C.; Lea, R. A.; Haupt, L. M.; Chambers, S. K.; Youl, P. H.; Griffiths, L. R. Association of the microRNA-Single Nucleotide Polymorphism rs2910164 in miR146a with sporadic breast cancer susceptibility: A case control study. Gene 2016, 576, 256-260.

8. Qi, J.-H.; Wang, J.; Chen, J.; Shen, F.; Huang, J.-T.; Sen, S.; Zhou, X.; Liu, S.-M.. Highresolution melting analysis reveals genetic polymorphisms in MicroRNAs confer hepatocellular carcinoma risk in Chinese patients. BMC Cancer 2014, 14, 643-655.

9. Zeng, Y.; Zhu, G.; Yang, X.; Cao, J.; Jing, Z.; Zhang, C. A quantum dot-based microRNA nanosensor for point mutation assays. Chemical Communications 2014, 50, 7160-7162.

10. Guo, Q.; Bai, Z.; Liu, Y.; Sun, Q. A molecular beacon microarray based on a quantum dot label for detecting single nucleotide polymorphisms. Biosensors and Bioelectronics 2016, 77, $107-110$.

11. Khoshfetrat, S. M.; A. Mehrgardi. M. Dual amplification of single nucleotide polymorphism detection using graphene oxide and nanoporous gold electrode platform. Analyst 2014, 139, 51925199 .

12. Park, K.; Choi, B.; Kim, Y. S.; Shin, S.; Hah, S. S.; Jung, W.; Oh, S.; Kim, D. Detection of single-base mutation in RNA using T4 RNA ligase-based nick-joining or DNAzyme-based nickgeneration. Analytical Biochemistry 2011, 414, 303-305. 
13. Yoon, H. R.; Lee, J. M.; Jung, J.; Lee, C.; Chung, B. H.; Jung, Y. Highly improved specificity for hybridization-based microRNA detection by controlled surface dissociation. Analyst 2014, $139,259-265$.

14. Peterson, A. W.; Wolf, L. K.; Georgiadis, R. M. Hybridization of Mismatched or Partially Matched DNA at Surfaces. J. Am. Chem. Soc. 2002, 124, 14601-14607.

15. Qavi, A. J.; Mysz, T. M.; Bailey, R. C. Isothermal Discrimination of Single-Nucleotide Polymorphisms via Real-Time Kinetic Desorption and Label-Free Detection of DNA Using Silicon Photonic Microring Resonator Arrays. Anal. Chem. 2011, 83, 6827-6833.

16. Naiser, T.; Kayser, J.; Mai, T.; Michel, W.; Ott, A.Position dependent mismatch discrimination on DNA microarrays - experiments and model. BMC Bioinformatics 2008, 9, 509-521.

17. Pan, S.; Sun, X.; Lee, J. K. Stability of complementary and mismatched DNA duplexes: Comparison and contrast in gas versus solution phases. International Journal of Mass Spectrometry 2006, 253, 238-248.

18. Squires, T. M.; Messinger, R. J.; Manalis, S. R. Making it stick: convection, reaction and diffusion in surface-based biosensors. Nat Biotech 2008, 26, 417-426.

19. Sun, H.; Xiang, J.; Zhang, X.; Chen, H.; Shi, Y.; Yu, L.; Yang, Q.; Li, Q.; Guan, A. A colorimetric temperature sensor of a cyanine dye supramolecule and its application in reversible switch. Applied Physics Letters 2014, 105, 071914.

20. Sanborn, M. E.; Connolly, B. K.; Gurunathan, K.; Levitus, M. Fluorescence Properties and Photophysics of the Sulfoindocyanine Cy3 Linked Covalently to DNA. J. Phys. Chem. B 2007, $111,11064-11074$. 
21. Vijayendran, R. A.; Ligler, F. S.; Leckband, D. E. A Computational Reaction-Diffusion Model for the Analysis of Transport-Limited Kinetics. Anal. Chem. 1999, 71, 5405-5412.

22. Wang, J.-Y.; Drlica, K. Modeling hybridization kinetics. Mathematical Biosciences 2003, 183, $37-47$.

23. You, Y.; Moreira, B. G.; Behlke, M. A.; Owczarzy, R. Design of LNA probes that improve mismatch discrimination. Nucleic Acids Res 2006, 34, e60.

24. Castoldi, M.; Schmidt, S.; Benes, V.; Noerholm, M.: Kulozik, A. Z.; Hentze, M. W.; Muckenthaler, M. U. A sensitive array for microRNA expression profiling (miChip) based on locked nucleic acids (LNA). RNA 2006, 12, 913-920.

25. Karberg, M.; Leavitt, R.; Cabaya, D. A.; Eden, M. V.; Jia, X. Y. Abstract \#5147: A method for quantifying DNA methylation percentage without chemical modification. Cancer Res 2009, $69,5147-5147$

26. Teerapanich, P.; Pugnière, M.; Henriquet, C.; Lin, Y.; Chou, C.; Leïchlé, T. Nanofluidic Fluorescence Microscopy (NFM) for real-time monitoring of protein binding kinetics and affinity studies. Biosensors and Bioelectronics 2017, 88, 25-33.

27. Wong, E. L. S.; Chow, E.; Gooding, J. J. DNA Recognition Interfaces: The Influence of Interfacial Design on the Efficiency and Kinetics of Hybridization. Langmuir 2005, 21, 69576965.

28. Leïchlé, T.; Lin, Y.; Chiang, P.; Hu, S.; Liao, K.; Chou, C. Biosensor-compatible encapsulation for pre-functionalized nanofluidic channels using asymmetric plasma treatment. Sensors and Actuators B: Chemical 2012, 161, 805-810. 
TOC

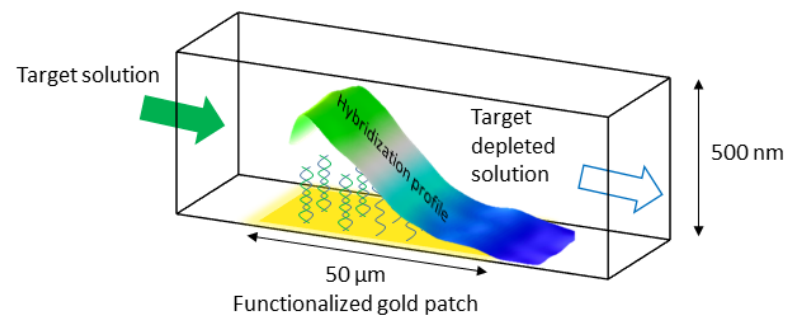

